\title{
REFORMA EDUCACIONAL CHILENA: UnA VISIÓN DE FUTURO
}

\author{
ANDRÉs TRONCOSO ÁVILA ${ }^{1}$
}

\section{Introducción}

La sociedad chilena enfrenta variados y significativos desafíos para convertirse en una sociedad verdaderamente desarrollada, y pueda así superar sus profundas desigualdades. EI desafío primordial y fundamental es la Educación. Además, de aquellos elementos asociados directamente a ganancias en productividad y equidad, un acceso igualitario a educación de calidad ayuda a configurar una sociedad con más oportunidades, más justa, que empodera a la ciudadanía en su quehacer cotidiano.

Chile ha conseguido importantes logros en materia educativa: cobertura, alta inversión en infraestructura, equipamiento y programas focalizados. Sin embargo, a pesar de los esfuerzos, la desigualdad educacional y la segregación continúan en niveles alarmantes y la Educación Pública en consecuencia se ha visto especialmente reducida y fragilizada.

Se dice que la educación chilena del siglo XXI debe estar al servicio de nobles ideales, de una sociedad fuertemente cohesionada, que supere la segregación social, que promueva la formación integral, la inclusión, la solidaridad y la democracia como valores centrales para el desarrollo nacional y de cada uno de los ciudadanos. El Estado debe asegurar que, independientemente del origen, condición, historia o lugar donde se habite, toda niña y niño tendrá el derecho de integrarse a un proceso educativo donde será reconocido, y con acceso a experiencias de aprendizaje que le permita desplegar talentos y lograr las competencias requeridas por la sociedad actual, promoviendo un desarrollo ciudadano a escala humana y basado en el bien común.

La gran deuda de hoy es mejorar en forma transversal la calidad de la educación, entendida integralmente y no sólo como el resultado de una serie de pruebas estandarizadas que

\footnotetext{
${ }^{1}$ Doctor en Educación por la Universidade Estadual de Campinas. Académico de la Universidad de Concepción Campus Los Ángeles - Concepción - Chile.
} 
se aplican en varios niveles y ámbitos educativos como también reposicionar a la Educación Pública en todas las etapas. Para que el Estado pueda entregar una mejor educación resulta imprescindible contar con un sistema más integrado y equitativo, que no deje como hoy a rezagados y excluidos. Ello significa, también, que un diseño adecuado no puede implicar una carga financiera insostenible ni para los estudiantes ni para sus familias.

El Estado, a través de la educación pública, pretende desarrollar un rol fundamental en cada nivel educacional, garantizando el ejercicio del derecho a una educación de calidad, entendida ésta como el potencial que posee cada persona y por lo tanto implica otorgarle a cada uno la posibilidad de un desarrollo integral profundo y así ir fortaleciendo de esta manera a la educación pública, entregando a su vez garantías explícitas y exigibles a los estudiantes. Para mejorar en consecuencia la educación de calidad, el Estado requerirá incrementar de un mayor gasto público permanente entre 1,5 y 2 puntos del PIB.

\section{Pilares Fundamentales}

Cuatro son los pilares que contemplan la reforma educativa chilena propuesta por el gobierno y que están referidas a: la calidad educativa; segregación e inclusión; gratuidad universal y fin al lucro en todo el sistema educativo.

a) El concepto de calidad educativa se ha visto empobrecido debido al uso y las consecuencias que se les ha dado a los resultados del Sistema de Medición de la Calidad de la Educación (SIMCE), y como producto de lo anterior se han promovido las prácticas tales como la selección de los alumnos, la exclusión de los que no rinden y el entrenamiento de pruebas, por lo tanto, no se ha venido desarrollando la capacidad de pensar. Hay que lograr superar el uso de las malas prácticas en el desarrollo del proceso de aprendizaje, impulsando una "comprensión más compleja e integral de calidad" para recuperar el sentido de la labor educativa.

Lo que se busca es que todos los establecimientos educacionales deberán ser de excelencia en el amplio sentido de la palabra y para que esto se cumpla, se deben entregar los instrumentos y recursos necesarios para mejorar la calidad y asegurar una efectiva fiscalización, como el debido apoyo y acompañamiento. Indudablemente que para llegar a la excelencia se requiere que la profesión docente esté dentro de las más valoradas socialmente y de las más exigentes, lo que no ocurre. 
Para poder mejorar el estatus del profesorado será necesario también conceder el espacio dentro de su contrato laboral y las herramientas necesarias para que los profesionales de la educación, directivos y asistentes de la educación puedan desarrollar su labor con mayor dignidad. Sin embargo, para mejorar la calidad y la excelencia de la educación se requiere que la formación del profesorado sea de un buen nivel y aquí la responsabilidad está en las Facultades y Escuelas formadoras de profesores las cuales deberán estar acreditadas por los organismos competentes y el ingreso a pedagogía supeditado con altas exigencias, siendo a su vez procesos más equitativos de acceso y compromisos para reducir la deserción en las carreras de educación. b) Será un compromiso reducir la segregación y contar con un país más integrado social, cultural e inclusivo, teniéndolo como objetivo transversal en el diseño de políticas públicas y no sólo lo educativo. Según los indicadores externos, Chile es el segundo país con más segregación social entre los países evaluados por el Program for International Student Assessment (PISA), evaluación a cargo de la OCDE.

Una realidad preocupante es que, según UNICEF, existen 300.000 niños y niñas que se encuentran fuera del sistema escolar y que en educación superior la deserción puede superar el $40 \%$ en algunas carreras, sobre todo en los sectores más vulnerables. Igualmente, grave es que, tras cada uno de estos jóvenes, hay muchos otros que también enfrentan dificultades pero que, a pesar de seguir en el sistema, quedan excluidos de los aprendizajes.

También es preocupación construir un sistema inclusivo siendo este un desafío muy grande, en donde uno de los objetivos principales será eliminar la selección al ingreso de las escuelas, lo que está ocurriendo en la actualidad. El principal propósito es lograr que el proceso educativo llegue a todos los estudiantes, les haga sentido y les motive en su propio desarrollo.

A nivel escolar, esto requiere una mirada sistémica y un trabajo continuo de las escuelas para lo cual los profesionales de la educación poseen muy débiles fundamentos y los profesores, en su mayoría, no están siendo preparados para manejar esta diversidad. Aún las escuelas no suelen desarrollar estrategias para abordarla, ni cuentan con el tiempo y los recursos adecuados para hacerlo.

c) Otro de los pilares que plantea la propuesta de reforma educacional es llegar a la gratuidad universal, lo que significa un cambio de paradigma. Lo que implica pasar de la educación como un bien de consumo que es posible transar en el mercado y la competencia como mecanismo 
regulador de la calidad, a un sistema educacional coordinado que ofrece a las niñas, niños y jóvenes de Chile un derecho social.

Es importante señalar que en Chile el nivel socioeconómico al nacer sigue determinando el futuro de las niñas y niños. Por tanto, el rol del Estado es lograr que el derecho a una educación de calidad no dependa de la capacidad de pago de las familias y que su nivel de ingreso o endeudamiento no determine el acceso a la educación y con ello su futuro. Lo que importa es disminuir la desigualdad la que supone que el nivel socioeconómico y cultural de las y los estudiantes no sea una barrera al acceso, por lo que el Estado debe avanzar decididamente hacia la gratuidad universal que en décadas pasadas funcionaba la educación gratuita.

d) El Fin al lucro en todo el sistema educativo es otro de los grandes pilares que considera esa propuesta de reforma. No se puede seguir en Chile que la educación siga dominada por la lógica de cuasi mercado, cuyo motor es la competencia por los alumnos, lo que ha reducido la participación del Estado en la provisión, limitado las mejoras en calidad y dificultado el resguardo de la educación como un derecho.

Se visualiza que el gran temor que tienen los sostenedores privados en relación a la propuesta del fin al lucro es que el Estado desea terminar con el sector particular subvencionado lo cual no es así, lo que desea el Estado es garantizar a los padres que éstos puedan elegir el modelo educativo que quieran para sus hijos. Por su parte el Estado seguirá respetando la existencia de un sistema mixto, pero debe hacer valer el derecho a una educación de calidad a cada niña y niño. Por tal razón, todos los recursos que el Estado destine a la educación deben traducirse en un mejoramiento de la calidad. Lo que se eliminará será la existencia de lucro con recursos públicos, por tanto, se dirigirá el financiamiento público únicamente a instituciones sin fines de lucro, que tengan como único fin la entrega de educación de calidad en el marco de un derecho social.

\section{Principios de la Reforma}

Entendida la educación como un derecho social, en la que todos los ciudadanos tienen la opción a educarse para que de esta manera puedan elegir con libertad sus caminos en la vida. Es por tal razón que la propuesta de reforma plantea una serie de principios y/o lineamientos que orienten esta nueva reforma que se presentan a continuación: 
- La educación es un derecho social fundamental teniendo un valor público innegable y está a la base de una sociedad justa, democrática y participativa. Nuestra sociedad debe abandonar la práctica que han permitido tratar la educación como un bien de consumo.

- La educación debe otorgar garantías explícitas para los ciudadanos en materia educativa, respecto de acceso, calidad y financiamiento.

- El Estado debe fortalecer su rol, como factor activo tanto en la entrega directa de servicios educativos, como en una estricta fiscalización del sistema, creando una Superintendencia de Educación que se preocupe del aporte que hace el Estado para con las Instituciones Educacionales y una Agencia de Calidad que se preocupe de la entrega del tipo de educación.

- Preocupación por el fortalecimiento de la educación pública como motor y sello del proceso de la Reforma Estructural, fijando los estándares de calidad y teniendo la presencia relevante en todo el territorio. Esa debe ser la base y herramienta más potente sobre la que se irá construyendo el proyecto educativo, la cohesión y la integración social.

- El sistema educativo será el encargado de promover la integración y la inclusión social en todos los niveles, no olvidando que las actuales reglas y normas que nos han conducido nos ubica en uno de los países cuyo sistema escolar es de los más segregados socialmente, para lo cual el Estado será el encargado activamente de abordar esta situación.

- El proceso educativo y los aprendizajes deben ser el centro de las políticas públicas. Por lo tanto, será necesario alinear el proceso educativo con los ciudadanos que deseamos formar, el país que soñamos y los desafíos de la enseñanza en todos sus niveles y especialmente la de los más vulnerables.

- La generación de igualdad de oportunidades educativas desde la cuna, con acciones compensatorias para estudiantes que, producto de las desigualdades pasadas, hoy están en desventaja. Las brechas educativas se manifiestan desde antes que las niñas y niños ingresen al colegio. Las políticas públicas, tanto en magnitud de los recursos invertidos como en el diseño de instrumentos, deben contribuir a reducir y eliminar las distancias sociales. 
- La Reforma Educacional tendrá un abordaje de carácter global, incluyendo los niveles Preescolar, Básico, Medio (Humanista Científico - Técnico Profesional) y la Educación Superior, de manera de integrar y cubrir todo el espectro de la educación.

\section{La Reforma de la Educación Parvularia}

La actual reforma educacional será en un futuro de mucha preocupación para con el Sistema de Protección Integral de la Infancia "Chile Crece Contigo" la que garantizará el acceso gratuito a sala cuna y jardín infantil de jornada extendida, o modalidad equivalente, a los niños y niñas de familias pertenecientes al $60 \%$ más vulnerable de la población. Se va a entender estas garantías con nuevas medidas que aseguren una mayor cobertura, especialmente para los niños y niñas menores de 4 años, aumentando la calidad y con un marco institucional consistente con esta tarea.

La cobertura es también una preocupación futura aumentando la incorporación al sistema preescolar de por lo menos 90 mil niños y niñas en el tramo de 0 a 2 años, construyéndose unas 4.500 nuevas salas cuna. En el caso de la población infantil de 2 a 4 años se van a incorporar aproximadamente 34 mil niños y niñas, lo que va a implicar 1.200 nuevas salas en jardines infantiles. Luego, en relación con la población de pre-kínder y kínder, la cobertura en la actualidad es casi completa, por lo que la propuesta sugiere avanzar hacia la obligatoriedad de pre-kínder. Cada una de éstas iniciativas con el transcurso del Gobierno se van a ir entregando los detalles de las diferentes localidades en que se implementarán las 500 nuevas salas cunas. Las políticas públicas en educación señalan que será asegurado el acceso a sala cuna y jardín infantil de jornada extendida a todo hijo e hija de madre trabajadora, junto con la opción de jornada extendida en pre-kínder y kínder.

A diferencia de las políticas públicas implementadas por los Gobiernos anteriores, en el actual se va a establecer estándares más exigentes para este nivel educacional, principalmente a través de mejorar el coeficiente técnico, es decir, más profesionales y técnicos por niño. Adicionalmente, se instalará un sistema de acreditación y de aseguramiento de la calidad educativa para todos los establecimientos de educación parvularia.

Para dar una institucionalidad a la Educación Parvularia, el Ministerio de Educación ha creado una Subsecretaría de Educación Parvularia, dependiente del Ministerio de Educación la 
cual tendrá el rol fiscalizador, ejercido por una Intendencia de Educación Parvularia en la Superintendencia de Educación, y del rol de provisión del servicio, a cargo de la Junta Nacional de Jardines Infantiles e Integra y otros prestadores de servicios debidamente acreditados.

Se dará, en consecuencia, un fuerte impulso en la formación y capacitación de educadores y educadoras, pues el Estado debe velar por la calidad de la formación de profesionales y técnicos que se desempeñan en este nivel educacional, implementándose una acreditación más exigente para las carreras respectivas y se desarrollarán programas de apoyo para su fortalecimiento. Con el avance del tiempo, se han ido desarrollando programas públicos especiales para adelantar en la capacitación y el perfeccionamiento de directoras, educadoras y técnicos de educación parvularia.

En lo específico se va a fortalecer el rol de las directoras y directores, capacitándoles en materia de gestión y administración de los Jardines Infantiles, fortaleciendo y actualizando el conocimiento de las educadoras y técnicas de educación parvularia, apuntando a mejorar las intermediaciones y acompañamiento en el desarrollo de niñas y niños, así como el trabajo con sus familias.

El compromiso de las políticas públicas educativas será la implementación de programas educacionales complementarios, que fortalezcan el rol de la familia como principal educadora de sus hijos e hijas, desde una perspectiva de desarrollo integral. Serán programas para niños y niñas que viven en ruralidad o en zonas de difícil acceso y, también, para aquellas familias cuya opción no es una sala cuna o jardín infantil convencional. Ejemplo de eso es la conformación de acciones educativas y recreativas para fines de semana. Particularmente importante será la atención integral en modalidades flexibles, para niños y niñas de comunidades indígenas entre 0 a 4 años. Finalmente, será implementada una política para hacer efectivo el derecho de las familias a participar y colaborar en el proceso educativo y en la toma de decisiones que conciernen a sus hijos, incorporando orientaciones, estrategias y acciones para asegurar un contacto regular entre los centros educativos y las familias.

\section{La Reforma a la Educación General}

Uno de los grandes desafíos será poner el Fin del Lucro en la Educación, lo que se tra- 
duce en que no se podrá lucrar con recursos públicos, por lo que el financiamiento será orientado exclusivamente a instituciones educacionales que sean sin fines de lucro, lo que se regulará y fiscalizará debidamente. Para implementar esa medida se tendrá que considerar los plazos y mecanismos que permitan a los establecimientos particulares subvencionados, que lo requieran, realizar los ajustes necesarios y adaptarse a las nuevas normas.

Otro de los aspectos que incluye la Reforma es el "Término del Financiamiento Compartido", para lo cual serán evaluados de manera rigurosa y periódica, llegando al monto real del costo que tiene una educación de calidad. Por tanto, sobre el resultado de ese análisis, la subvención estatal se irá gradualmente aumentando y el aporte de pago por parte de los apoderados disminuyendo hasta ser completamente eliminado con el transcurso del tiempo. Mientras dure el proceso de transición no se producirán nuevos incrementos a los límites de financiamiento compartido y los establecimientos no podrán elevar este cobro. El fin del financiamiento compartido, junto con otros mecanismos de selección permitirá terminar con la segregación social en la educación financiada con recursos públicos.

También la Reforma Educacional contempla el Término de la Municipalización, cuya implementación fue realizada en el año 1981, para lo cual se va a crear una nueva institucionalidad para la oferta pública de la educación general, terminando con la administración municipal. Esa nueva institucionalidad se prevé va a considerar los avances alcanzados respecto de la participación y tuición del mundo local en la educación. Desde el traspaso de la educación a los municipios se le generó a éstas un grado de complejidad tal que éstos nunca estuvieron preparados para recibir esta nueva función como lo fue la educación a los municipios. Hasta nuestros días presentan dificultades, salvo algunas excepciones que pudieron gestionar de mejor forma.

Adicionalmente, la educación municipal está sujeta a un conjunto de regulaciones y restricciones en cuanto a su gestión que inciden en su desempeño y, particularmente, en la gestión de los recursos humanos, por lo tanto, la administración de los establecimientos de educación pública debe volver a estar en manos de instituciones especializadas. Es así como la administración de los establecimientos municipales será reemplazada por una nueva institucionalidad pública, cuya estructura contempla la creación de un Servicio Nacional de Educación Pública descentralizado y Servicios Locales de Educación Pública, que mantengan la cercanía con la comunidad educativa. Ambas entidades estarán relacionadas a través del Ministerio de Educación. 
Es así que los establecimientos educacionales pasarán a depender de los Servicios Locales de Educación Pública. Éstos se definirán geográficamente según distritos escolares. El Servicio Nacional de Educación Pública tendrá por función principal el apoyo técnico pedagógico, administrativo y financiero de los establecimientos públicos, a través de los Servicios Locales.

Cada Servicio Local contará con personalidad jurídica y patrimonio propio y tendrá un Consejo Consultivo integrado por miembros de la comunidad educativa y representantes de las Facultades de Educación de instituciones públicas de la región donde se sitúa el distrito escolar. En lo inmediato, se está formulando un plan de acción de corto plazo orientado a recuperar los establecimientos con mayores deficiencias en materias técnico-pedagógicas y asumir el desafío de recuperar matrícula en la educación pública.

Otro de los aspectos considerados en esa reforma educativa tiene que ver con el término de toda forma de selección de los alumnos y alumnas a nivel de la escuela, tanto económica, social, académica y conductual. Adicionalmente, los establecimientos deberán considerar en su proyecto educativo la inclusión de niños con necesidades educativas especiales y la habilitación para que ello ocurra de manera adecuada.

Esa Reforma Educacional debe comenzar con la participación activa de todos los docentes, directivos y el personal asistente de la educación, garantizando a todas y todos los docentes de la educación pública la oportunidad de participar cada 4 años, de manera gratuita, en un programa de perfeccionamiento docente, ampliando la oferta y mejora de la calidad de las acciones de formación continua. Se va a constituir en cada región del país un Comité Regional de Desarrollo Profesional Docente, cuya función será planificar y coordinar acciones de formación continua adecuadas a las necesidades regionales, desarrollando programas de pos títulos por disciplinas para profesores de segundo ciclo y de formación general para el primer ciclo. Se va a potenciar la existencia de la Red de Maestros y retomando la Formación de Profesores Mentores para el apoyo e inducción a docentes principiantes.

\section{Formación de los Profesores}

En cuanto a la formación de profesionales de la educación y muy especialmente a los que asumen cargos directivos se desarrollará un programa de inducción para directores que 
asumen sus cargos por primera vez. Además de eso habrá la formación de una Escuela de Directivos para realizar capacitaciones periódicas, tendientes a fortalecer el liderazgo directivo con énfasis en la gestión administrativa y pedagógica que promueva la conformación de equipos de trabajo y de comunidades educativas.

La formación inicial será mejorada sugiriendo que sean las Universidades las que impulsen programas de reformulación curricular, siendo éstas las que promuevan el desarrollo de capacidades de los equipos académicos de las instituciones formadoras mediante programas de intercambio a nivel internacional y la utilización de becas de postgrado; estableciendo mayores exigencias para la acreditación de las carreras de pedagogía e instituir el carácter obligatorio y habilitante de la evaluación de egreso, aumentando los recursos públicos para fomentar la investigación en temas pedagógicos. La preocupación en cuanto a la formación del profesorado será contar con Escuelas de Pedagogía de Excelencia, para lo cual serán desarrolladas y fortalecidos convenios de gestión administrativa directiva y docente.

Otro de los aspectos importantes propuestos por la reforma educacional en desarrollo es el mejoramiento de una carrera docente que, entre otros aspectos va a definir mejores remuneraciones; introducción de incentivos y aumento gradualmente del número de horas de la jornada laboral docente que se destine a trabajo fuera del aula. La carrera docente deberá instalar el desarrollo profesional y el desempeño de calidad como elemento central de la carrera, estableciendo la reducción gradual del número máximo de estudiantes por curso en la educación subvencionada.

En cuanto a la Educación Técnico Profesional dos serán los problemas fundamentales que deberán enfrentarse: su actual desvinculación con una trayectoria de formación profesional que incluya la educación media, superior y procesos de capacitación con su consiguiente falta de pertinencia respecto de los requerimientos del mundo laboral, sumándose a esto los altos niveles de deserción y baja titulación oportuna que presenta el sector de la educación técnico profesional. La consolidación de un sistema de formación diversificado que permita que todos los y las jóvenes tengan un proyecto de vida y puedan seguir una trayectoria educativa laboral de acuerdo a sus preferencias y talentos, independientemente de su nivel socioeconómico. Es en esa modalidad educativa donde se requieren instalar los mayores esfuerzos para lograr la excelencia en todos los establecimientos.

En cuanto a las Mallas Curriculares de la Educación Media Técnico Profesional, serán 
rediseñadas de manera tal que exista la posibilidad real de hacer una trayectoria conducente a títulos técnicos y profesionales superiores, creando en cada región del país un Centro de Entrenamiento de alto nivel para ofrecer formación práctica a los estudiantes, consistente con la vocación productiva de la región respectiva. Cada región del país contará con un Consejo de Formación Técnico Profesional, de carácter público-privado, que velará por la articulación completa del sistema dando lineamientos para el desarrollo de especialidades en la región, tanto a nivel medio como superior.

\section{La Reforma de la Educación Superior}

Otro aspecto que considera la reforma educacional es la Educación Superior y es en este nivel que los estudiantes junto a sus familias enfrentan dos tipos de problemas. En primer lugar, es el acceso, ya que existen barreras de financiamiento y académicas. Ambas son particularmente relevantes y discriminatorias en el caso de estudiantes pertenecientes a grupos en situación de mayor vulnerabilidad económica. En segundo lugar, por la existencia de serias dificultades en el control de la calidad de las instituciones de educación superior, cuestión que se ve agudizada por la baja fiscalización de los Institutos Profesionales y los Centros de Formación Técnica, para el cual será desarrollada una nueva institucionalidad que permita recuperar la confianza en el sistema y sus instituciones y que pueda dar respuesta a los desafíos del país en los ámbitos del desarrollo productivo, científico y cultural.

En consecuencia, la educación superior debe ser un derecho social efectivo, estableciendo garantías explícitas para los ciudadanos en materia de educación superior, tanto de acceso como de calidad y financiamiento. Esas garantías darán un trato preferente a los estudiantes de menores ingresos. Sin embargo, para cumplir con dichas garantías, será necesario tener un Estado activo tanto en la entrega directa de servicios educativos como en la estricta fiscalización de los oferentes, tarea que tendrá especial dedicación y rapidez.

Otro de los desafíos de la educación superior será avanzar en la gratuidad universal y efectiva, cuyo proceso se prevé tomará a lo menos 6 años. Durante el próximo período de Gobierno se verá implementada la gratuidad de al menos el 70\% de los estudiantes pertenecientes al grupo más vulnerable de la población, abarcando extensamente a la clase media. Dicha gratuidad podrá ser ejercida en toda institución de educación superior que cumpla, entre otros aspectos, con: estar acreditada; acatar estrictamente las normas que no permiten la existencia 
de lucro; estar incorporada a las nuevas normas sobre acceso de equidad; desarrollar programas de reforzamiento y acompañamiento para los alumnos vulnerables, y que se rija por el sistema de aranceles que determine el Ministerio de Educación. Para la fijación de los aranceles se creará una nueva institucionalidad, que considerará un grupo de expertos que determinarán y revisarán periódicamente el monto de dichos aranceles, siendo reguladas por las autoridades gubernamentales.

Preocupación fundamental será la modernización de la institucionalidad pública de la educación superior, creando la Subsecretaría de Educación Superior; teniendo esta competencias claras y recursos necesarios para cumplir sus funciones, en especial, la fiscalización del uso de los recursos públicos, velar que se cumpla la prohibición de lucro, y la Agencia de la Calidad de la Educación Superior, responsable de la acreditación y de velar por la calidad de la educación superior. Esa nueva institucionalidad permitirá una relación más directa y productiva entre el mundo de la educación superior y las autoridades del sector.

También será creado un Programa para Incrementar la Equidad en el Acceso a la Educación Superior, que contrapese el sesgo socioeconómico existente en los requisitos académicos. Para ello, las instituciones de educación superior que opten por recibir financiamiento público deberán suscribir un convenio de deberes y derechos con el Ministerio de Educación para aplicar este nuevo mecanismo de acceso. Ese mecanismo consistirá en establecer "cupos de equidad", adicionales al sistema regular, equivalente al menos a un $20 \%$ de la matrícula de cada una de las carreras que ofrezca la institución para estudiantes pertenecientes al $40 \%$ más vulnerable.

Como parte del Programa para Incrementar la Equidad en el Acceso a la Educación Superior, se va a implementar un programa permanente de acciones de apoyo a los estudiantes vulnerables, con el propósito de evitar la deserción de los estudios superiores. Eso incluye programas especiales en los establecimientos de enseñanza media para preparar a los jóvenes; involucrar a sus familias; apoyar y desarrollar competencias en los establecimientos de educación superior; y reformar el Aporte Fiscal Indirecto (AFI) para que incentive la matrícula y retención de estudiantes en situación de vulnerabilidad. En cuanto a los sistemas de acreditación, se va a establecer un nuevo sistema de acreditación de instituciones de educación superior, que va a considerar la acreditación tanto institucional como por carreras, la que será obligatoria para todas las instituciones de educación superior (Universidades, Institutos Profesionales y Centros 
de Formación Técnica).

La nueva reforma va a establecer un Nuevo Fondo de Apoyo a la Investigación Universitaria, de una magnitud suficiente para que las instituciones no busquen cargar en los aranceles la labor que realizan en materia de investigación. Ese fondo estará disponible para todas las universidades públicas, y para todas aquellas que acrediten capacidades de investigación y firmen convenios de desempeño con el Ministerio de Educación. Para dar cumplimiento a ese esfuerzo financiero se va a establecer un trato preferente con las universidades de propiedad del Estado. Esas universidades deberán introducir las modificaciones necesarias en su gestión, para rendir cuentas respecto de sus planes de desarrollo y del financiamiento obtenido. Ese nuevo trato incluye un fondo de aporte basal permanente exclusivo para las universidades estatales y un fondo permanente de apoyo a las universidades estatales regionales.

Tal como lo concibe la reforma educacional en cuanto a la educación superior ya fueron creadas dos nuevas universidades estatales regionales donde la presencia del Estado en cuanto a educación superior no existía. Esas Universidades fueron instaladas en la Región de Aysén, al sur del país y en la Región de O'Higgins, zona Central, las cuales comenzarán con la admisión de alumnos en marzo del año 2017.

Al alero de algunas universidades serán creados Centros de Formación Técnica públicos, que tendrán presencia en todas las regiones, cada Centro estará vinculado a una universidad pública. Aspirando a que esas instituciones marquen la pauta en cuanto a los estándares esperados de calidad en la educación técnica con una fuerte identificación y vinculación con el desarrollo productivo regional.

Finalmente, muchas de las acciones manifestadas por las autoridades superiores de gobierno están siendo implementadas. Las que requieren ser motivo de normas legales las están resolviendo a nivel de las comisiones de educación para posteriormente enviarlas al Parlamento para transformarlas en Leyes Oficiales.

\section{Textos de apoyo}

AGENCIA DE CALIDAD DE LA EDUCACIÓN. Informe Nacional. Resultados Chile. PISA 2012.

AGUILA, Ernesto. Del Estado Docente al Estado subsidiario: de la escuela pública selectiva a la escuela segregada. Ponencia en Cátedra Foucault, 2013. 
ATRIA, F. ¿Qué educación es "pública"?. In: BELLEI, C.; CONTRERAS, D.; VALENZUELA, J. P., Ecos de la revolución pingüina. Avances, debates y silencios en la reforma educacional. Santiago Pehuén: Universidad de Chile-UNICEF, 2010. p. 31-50.

GOBIERNO DE CHILE. Proyecto de Ley de Sistema Nacional de Educación Pública. 02 de Noviembre de 2015. Santiago - Chile.

MINEDUC - Ley 20.903. Crea el Sistema de Desarrollo Profesional Docente. Santiago. Chile.

MINEDUC (2015) Ley № 20.845. Inclusión Escolar que regula la admisión de los y las estudiantes, elimina el financiamiento compartido y prohíbe el lucro en establecimientos educacionales que reciben aportes del Estado. Santiago. Chile.

PRESIDENCIA DE LA REPÚBLICA. Mensaje de S.E. La presidenta de la República con el que inicia un proyecto de Ley que crea el Sistema de Educación Pública y modifica otros cuerpos legales. Santiago 02 de Noviembre de 2015. 